Radiologe 2020 · 60 (Suppl 1):S41-S53 https://doi.org/10.1007/s00117-020-00696-0 Published online: 19 May 2020

(c) Springer Medizin Verlag $\mathrm{GmbH}$, ein Teil von Springer Nature 2020

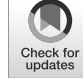

\author{
B. Theek ${ }^{1,2} \cdot$ T. Nolte $^{1} \cdot$ D. Pantke ${ }^{1}$ F. Schrank ${ }^{1} \cdot$ F. Gremse ${ }^{1}$. V. Schulz ${ }^{1,2,3}$. \\ F. Kiessling ${ }^{1,2,3}$ \\ 'Institute for Experimental Molecular Imaging, Medical Faculty, RWTH Aachen International University, \\ Aachen, Germany \\ ${ }^{2}$ Fraunhofer Institute for Digital Medicine MEVIS, Bremen, Germany \\ ${ }^{3}$ Comprehensive Diagnostic Center Aachen (CDCA), University Hospital RWTH Aachen, Aachen, Germany
}

\title{
Emerging methods in radiology
}

\section{Several milestones can be high- lighted in the development of imaging methods. It started with the detection of X-rays, enabling the first noninvasive view into the body, followed by the introduction of to- mographic methods, providing more anatomical details, and the intro- duction of functional and molecular imaging, paving the way for a spa- tially resolved pathophysiological characterization.}

Imaging methods and probes continue to undergo advances, and new imaging methods are emerging, increasing the spectrum of accessible biomedical features. However, with the rise of deep learning, radiomics, and comprehensive data analysis, a new era of diagnostics starts, where image features from various sources can be integrated and interpreted in concert with other diagnostic data. This article highlights some of the most recent developments in imaging technology, considering the opportunities ushered in with advanced digital data analysis. However, because of the limited space in this article, we only focus on approaches that are already close to or in the process of clinical translation.

\section{Advances in computed tomography}

Due to concerns regarding the radiation risk of computed tomography (CT), intense research has been conducted on the processing of low-dose CT data. Iterative reconstruction techniques achieve higher image quality than filtered backprojection, but reconstructed images from lowdose CT data still suffer from increased noise and artifacts. Recent publications present deep-learning methods from computer vision to improve the quality of medical images. Convolutional neural networks show promising results for low-dose CT denoising in the form of residual autoencoders ( $\bullet$ Fig. 1a-c; [12]) and generative adversarial networks. Furthermore, neural networks can be used to reduce metal artifacts [27] and to optimize iterative reconstruction algorithms [71].

Advances in CT hardware have been achieved with smaller detector element sizes, resulting in ultra-high-resolution CT scanners, which use matrix sizes of up to $2048 \times 2048$ pixels ( $\bullet$ Fig. $1 \mathrm{~d}-\mathbf{i}$; [26]).

\section{Photon-counting computed tomography}

Another CT development gaining clinical interest is photon-counting CT (PCCT), which uses energy-resolving detectors and thus enables the acquisition of $\mathrm{CT}$ scans at multiple energies. Initial evaluations have shown its capability of highresolution imaging with reduced radiation dose [56], as well as the distinction between different tissue types and contrast agents $[48,53]$. One-step inversion methods have been recently introduced for the joint material decomposition and tomographic reconstruction of PCCT data, which can improve image quality and decrease noise levels compared with sequential approaches [40]. Clinical PCCT systems are being developed but face several technical challenges, including pile up and cross talk in detector elements.

\section{Phase-contrast imaging}

Another promising innovation in the field of X-ray imaging is phase-contrast imaging (PCI). While standard X-ray systems image the absorption of photons passing through a patient, here image contrast is generated by the phase shift of X-rays, caused by different refractive indices of tissues. Talbot-Lau interferometers that use fine gratings in the X-ray beam path enable the reconstruction of three different images: the typical absorption image, a differential phase image, and a dark-field image. The latter measures ultra-small angle scattering caused by inhomogeneities in materials at the micrometer scale. Use cases for X-ray dark-field imaging include the detection and quantification of pulmonary emphysema and imaging of brain connectivity $[68,69]$. Furthermore, PCI can improve the sensitivity of calcification classification in breast imaging, and clinical systems in the field of mammography are being developed [51].

\section{Advances in magnetic resonance imaging}

Magnetic resonance imaging (MRI) is known for its excellent soft tissue contrast, which is based on nuclear magnetic resonance of (proton) spins without the need for ionizing radiation. Throughout the history of MRI, continuous efforts were made to accelerate the time-intensive examinations. After multichannel receive arrays enabled parallel imaging with SENSE or GRAPPA reconstruction, the combination with k-t-space imaging further improved the temporal resolution 

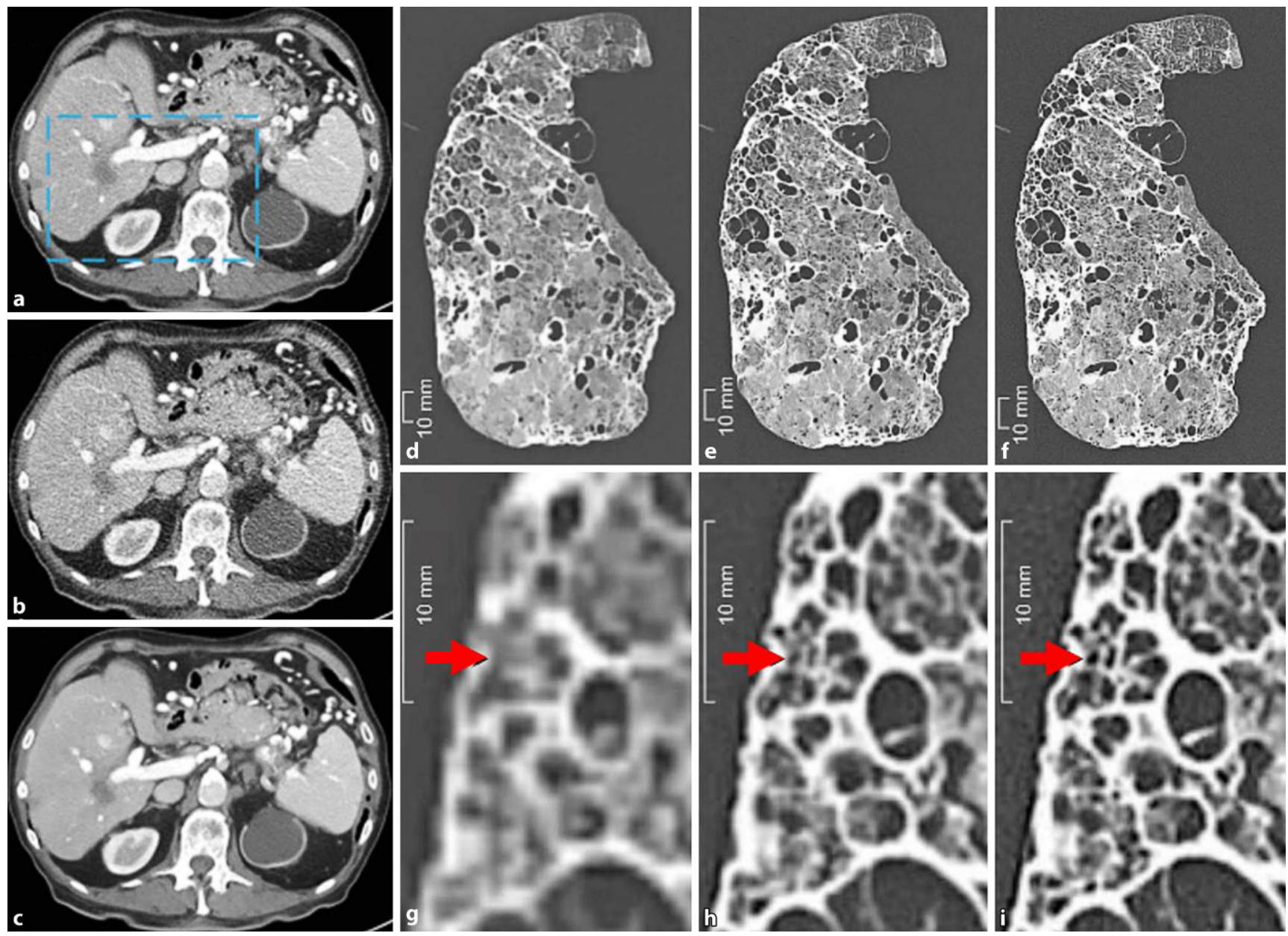

Fig. $1 \Delta$ a-c Denoising results using deep learning from an abdominal image with a metastasis in the liver. a Normal-dose computed tomography (CT), b low-dose CT, and c residual encoder-decoder CNN (RED-CNN). The RED-CNN suppresses noise while preserving the image structure. (Reprinted with permission from [12]). d-i Ultra-high-resolution CT images of a cadaveric lung with honeycombing using $512 \times 512$ (d), $1024 \times 1024$ (e), and $2048 \times 2048$ (f) matrix sizes and the corresponding images that were magnified sixfold $(\mathbf{g}-\mathbf{i})$. The small honeycombing air spaces are depicted more clearly in the $2048 \times 2048$ matrix than in the $1024 \times 1024$ matrix (arrow); the $512 \times 512$ matrix was the most blurred and could not depict some air spaces. Image noise was visually lower in the following order: $512 \times 512,1024 \times 1024$, and $2048 \times 2048$. (Reprinted with permission from [26])

of dynamic scans. Simultaneous multislice imaging combined parallel imaging along the slice direction with the use of multi-band RF pulses, thereby permitting the acquisition of multiple slices at a time with minor penalty on the signalto-noise ratio. The use of non-Cartesian data acquisition schemes such as radial sampling enabled three-dimensional coverage of the heart with reduced motion sensitivity and, as a major breakthrough, real-time (and non-triggered!) imaging of the heart as well as the visualization of speech [58].

\section{Compressed sensing}

Compressed sensing (CS), introduced to the field of MRI research by Lustig et al. in 2007 , reconstructs the missing samples in incoherently undersampled MRI data in an iterative fashion, exploiting data sparsity. The basic principle of accelerated MRI is explained in - Fig. 2. In 2017, CS achieved FDA approval and enabled accelerated dynamic and volumetric imaging in many applications relying on speed, as, for example, in cardiac MRI, dynamic contrast-enhanced acquisitions, angiography, and pediatric imaging among others. For example, CS in conjunction with 4D-flow MRI made it possible to examine different aortopathies within a clinically feasible scan time [44].

While diagnostic MRI relies to a major extent on contrast-weighted images, faster scanning techniques in conjunction with model-based reconstruction techniques, such as MR fingerprinting [37], have started to enable a more widespread access to multi-parametric and quantitative tissue parameter measurements.

\section{Chemical exchange saturation transfer}

Recently, concerns were raised regarding the retention of gadolinium-based con- 
trast agents in the brain. As a first step toward gadolinium-free MRI examinations, reductions in the contrast agent dose may be feasible for certain applications [16]. Moreover, potential alternatives to gadolinium contrast agent-enhanced scans are increasingly explored in the clinical context. For instance, diffusion-weighted imaging provides an image contrast that is mediated by the diffusion processes of water molecules within tissue microstructures. In addition, chemical exchange saturation transfer (CEST) enables imaging of exogenous and, especially, of endogenous molecules such as amides, amines, glutamate, and creatine and thus has potential for imaging of metabolic processes. Applications of CEST comprise imaging of cerebral ischemia, neurological disorders, lymphedema, osteoarthritis, muscle physiology, and solid tumors $[29,62]$. The improved performance of CEST at high field strengths (i.e., min. $7 \mathrm{~T}$ for most metabolites) limits its widespread clinical application, although initial experiences with glucoCEST and gagCEST imaging at $3 \mathrm{~T}$ have been reported for tumor, brain, and cartilage imaging [33]. Moreover, the dependence of CEST contrast on a variety of experimental parameters constitutes a challenge for the reproducibility of CEST results [70].

\section{${ }^{13} \mathrm{C}$ hyperpolarized magnetic resonance imaging}

Another promising approach toward metabolic imaging is ${ }^{13} \mathrm{C}$ hyperpolarized MRI, for which a special preparation step results in highly increased signal from ${ }^{13} \mathrm{C}$ contrast agents. Among these, $\left[{ }^{1-13} \mathrm{C}\right]$ pyruvate is evaluated in clinical trials for the visualization of metabolic changes during treatment of prostate cancer (- Fig. 2a; [2]), brain cancer, as well as in cardiac diseases. Preclinical studies, moreover, showed potential for monitoring of liver and kidney disease and of inflammatory processes and investigated a variety of probes. In contrast to CEST, ${ }^{13} \mathrm{C}$ hyperpolarized MRI requires dedicated dual-tuned, multichannel coil arrays and a polarizer for probe delivery, which are nonclinical standards. Yet, one clinically approved polarizer (GE

Radiologe 2020 - 60 (Suppl 1):S41-S53 https://doi.org/10.1007/s00117-020-00696-0

(c) Springer Medizin Verlag GmbH, ein Teil von Springer Nature 2020

\section{B. Theek · T. Nolte - D. Pantke · F. Schrank · F. Gremse · V. Schulz · F. Kiessling}

\section{Emerging methods in radiology}

\section{Abstract}

Imaging modalities have developed rapidly in recent decades. In addition to improved resolution as well as whole-body and faster image acquisition, the possibilities of functional and molecular examination of tissue pathophysiology have had a decisive influence on imaging diagnostics and provided ground-breaking knowledge. Many promising approaches are currently being pursued to increase the application area of devices and contrast media and to improve their sensitivity and quantitative informative value. These are complemented by new methods of data processing, multiparametric data analysis, and integrated diagnostics. The aim of this article is to provide an overview of technological innovations that will enrich clinical imaging in the future, and to highlight the resultant diagnostic options. These relate to the established imaging methods such as CT, MRI, ultrasound,
PET, and SPECT but also to new methods such as magnetic particle imaging (MPI), optical imaging, and photoacoustics. In addition, approaches to radiomic image evaluation are explained and the chances and difficulties for their broad clinical introduction are discussed. The potential of imaging to describe pathophysiological relationships in ever increasing detail, both at whole-body and tissue level, can in future be used to better understand the mechanistic effect of drugs, to preselect patients to therapies, and to improve monitoring of therapy success. Consequently, the use of interdisciplinary integrated diagnostics will greatly change and enrich the profession of radiologists.

\section{Keywords}

Molecular imaging · Radiomics - Biomarker . Deep learning $\cdot$ Diagnostic imaging

\section{Neue technische Entwicklungen in der Radiologie}

\section{Zusammenfassung}

Die bildgebenden Verfahren haben sich in den letzten Jahrzehnten rasant weiterentwickelt. Neben verbessertem Auflösungsvermögen, Ganzkörpererfassung und schnellerer Bilderstellung haben vor allem die Möglichkeiten der funktionellen und molekularen Untersuchung der Gewebepathophysiologie die bildgebende Diagnostik maßgeblich beeinflusst und wegweisende Erkenntnisse geliefert. Viele aussichtsreiche Ansätze werden derzeit verfolgt, um das Spektrum der Anwendbarkeit von Geräten und Kontrastmitteln zu erhöhen und deren Sensitivität und quantitative Aussagekraft zu verbessern. Hinzu kommen neuartige Verfahren der Datenverarbeitung, der multiparametrischen Datenanalyse und der integrierten Diagnostik. Ziel dieses Beitrags ist es, einen Überblick zu geben, welche technologischen Neuerungen die klinische Bildgebung in Zukunft bereichern werden und welche diagnostischen Möglichkeiten daraus resultieren. Diese betreffen sowohl etablierte Bildgebungsverfahren wie Computertomographie, Magnetresonanztomographie, Ultraschall, Positronenemissionstomographie und "single photon emission computed tomography" als auch neue Verfahren wie "magnetic particle imaging", die optische Bildgebung und die Photoakustik. Ferner werden Ansätze der Radiomics-basierten Bildauswertung erläutert und die Möglichkeiten und Schwierigkeiten bei deren breiter klinischer Einführung diskutiert. Das Potenzial der Bildgebung, pathophysiologische Zusammenhänge in stetig steigendem Detailgrad sowohl auf Ganzkörperniveau als auch auf Gewebeebene zu beschreiben, kann in Zukunft genutzt werden, um die Wirkungsweise von Medikamenten besser zu verstehen, Patienten für Therapien gezielt auszuwählen und die Überwachung der Therapieerfolge zu verbessern. In der Folge wird der Einsatz fachübergreifender integrierter Diagnostik das Berufsbild des Radiologen stark verändern und bereichern.

Schlüsselwörter

Molekulare Bildgebung · Radiomics . Biomarker - Deep Learning - Diagnostische Bildgebung 


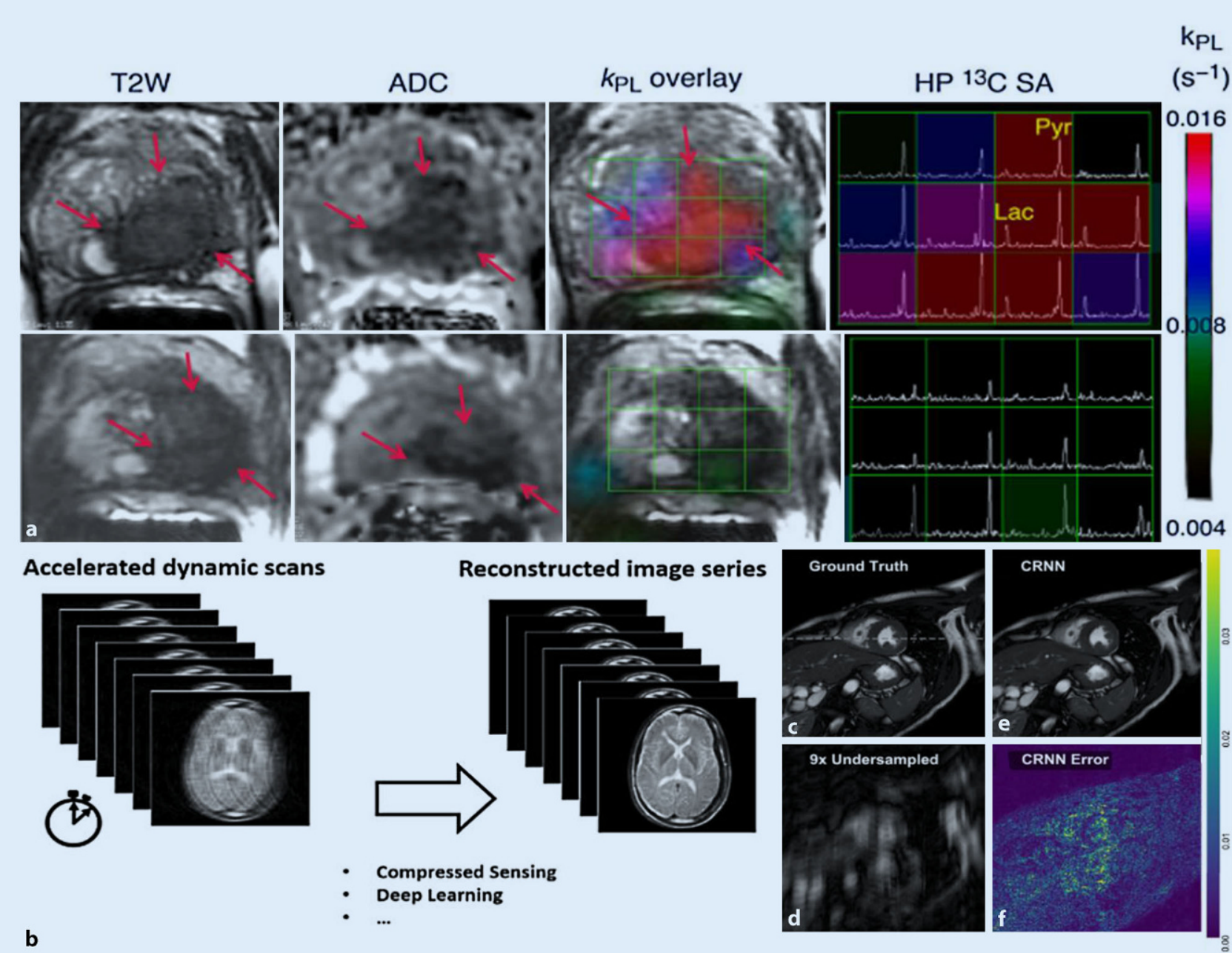

Fig. $2 \Delta \mathrm{a}^{13} \mathrm{C}$ hyperpolarized magnetic resonance imaging (MRI) as an alternative method to gadolinium-based imaging. From left to right: axial T2-weighted ( $T 2 W$ image of a 52-year-old prostate cancer patient with extensive high-grade prostate cancer, the water apparent diffusion coefficient map $(A D C)$, and an overlay of the pyruvate-to-lactate metabolic flux $(k P L)$ image with the corresponding hyperpolarized $(H P)^{13} \mathrm{C}$ array of spectra $(S A)$ are shown. Top row: before therapy; bottom row: 6 weeks after initiation of androgen ablation and chemotherapy. The kPL image indicates therapy response most clearly, corroborating the clinical response of the patient. (Reprinted with permission from [2]). b-f Compressed sensing and other emerging reconstruction methods enable faster MRI scanning. b Basic principle is demonstrated for a dynamic image series. Accelerated scanning is accomplished with undersampling, i.e., by gathering fewer data than needed for high-quality images. After performing a sophisticated reconstruction, high image quality is restored.c-f A deep-learning-based reconstruction method working with convolutional recurrent neural networks (CRNN) is applied to a cardiac MRI scan in axial view. While the undersampled image (d) is diagnostically unusable, the CRNN reconstruction (e) closely resembles the ground truth image (c), with a deviation of less than $3 \%$ as depicted in the colormap (f). (Reprinted with permission from [49])

SpinLab) is available on the market, encouraging a further translation of this technique.

\section{Deep-learning applications}

Recently, deep learning has had its advent in MRI with applications along the entire imaging chain [36]. Qin et al. presented an accelerated dynamic reconstruction using convolutional recurrent neural networks (CRNN) for undersampled car- diac MRI data [49]. Figure $2 \mathbf{c}-\mathbf{f}$ demonstrates that their deep-learning reconstruction closely resembles the ground truth dataset. Chen et al. applied a variational network to real-time reconstruction of undersampled abdominal MRI data [11]. Again, the deep-learning approach outperformed the CS reconstruction in speed and image quality. Zhu et al. generalized deep-learning-based image reconstruction to different sampling strategies and even to the recon- struction of PET raw data [74]. Deep learning was equally applied to quantitative MRI, for example, to accelerate MR fingerprinting reconstructions, to quantitative susceptibility mapping, and to estimate tissue microstructure properties from a reduced number of diffusion-weighted images [36]. Further applications of deep learning to MRI include image denoising and artifact detection, super-resolution, the generation of synthetic images, and image registra- 


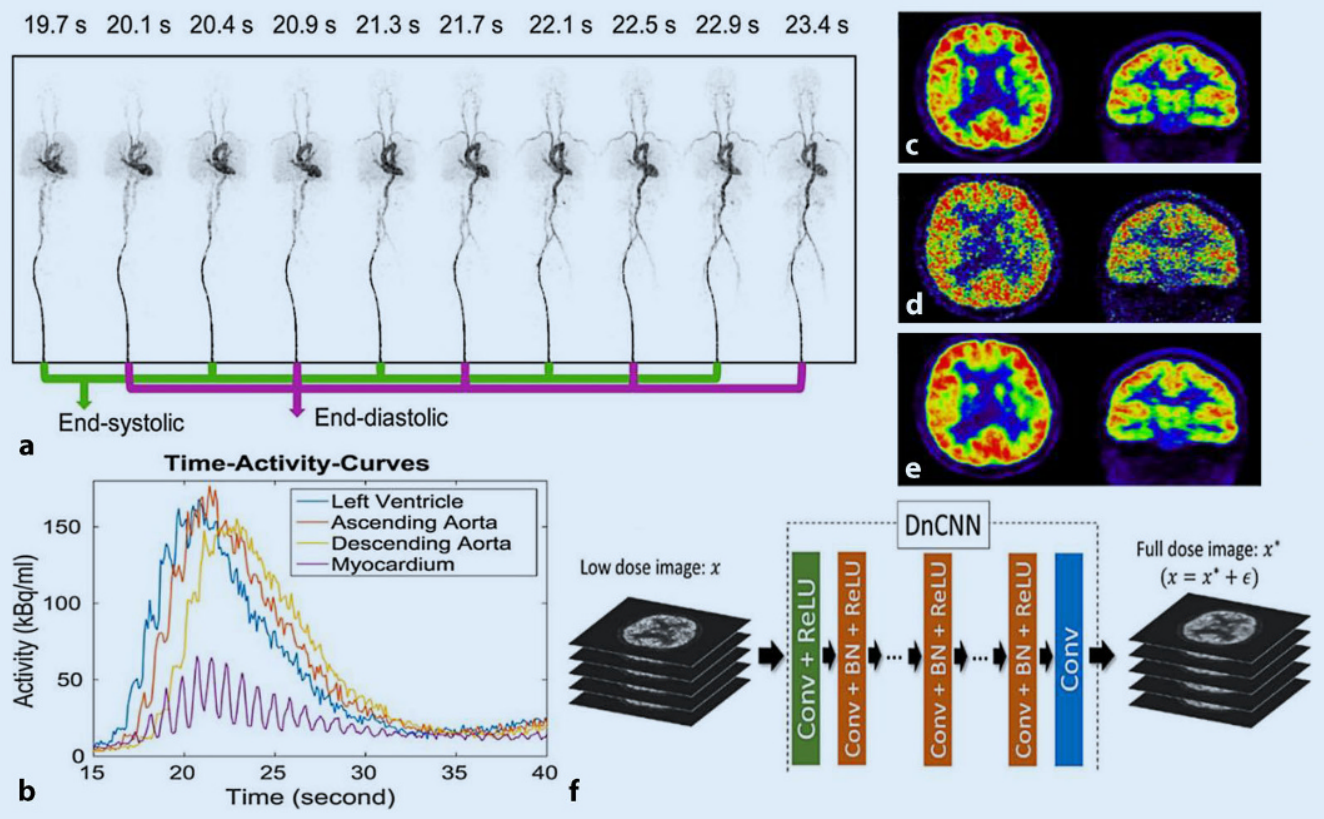

Fig. $3 \Delta$ a Reconstructed dynamic positron emission tomography (PET) images from the uEXPLORER total-body PET system. The images show frames of $100 \mathrm{~ms}$, alternating between the end-diastolic and end-systolic phases of five cardiac cycles. b Dynamic time-activity curves corresponding to a, showing the cardiac modulation of the tracer at different locations. (Reprinted with permission from [72]). c-e Results of a trained denoising convolutional neural network (DnCNN) combined with an iterative PET reconstruction demonstrating reconstructed PET images from low count statistics. c Full-dose image reconstructed with a reference reconstruction (OPOSEM method), $\mathbf{d} 10 \times$ low-dose OPOSEM image, and e $10 \times$ low-dose OPOSEM image with the proposed DnCNN method. $f$ Network topology of the DnCNN. (Reprinted with permission from [32])

tion [36]. Numerous studies demonstrate deep learning in disease prediction and diagnosis [36]. It is expected that many current research projects employing deep learning will become integrated in clinical products in the near future, although issues of data access and privacy need to be considered carefully.

\section{Advances in nuclear imaging techniques}

When the combination of positron emission tomography (PET) and computed tomography (CT) was introduced in 2000, commercial PET/CT and SPECT/CT systems used vacuum photomultiplier tubes (PMTs). However, semiconductor detectors (so-called silicon photomultiplier [SiPM]) were invented as early as in the 1990s, which revolutionized the entire field of nuclear medicine by surpassing traditional PMTs in aspects such as compactness, flexibility, and performance. Research groups have demonstrated excellent performance with benchtop and prototype systems, indicating further growth potential for this technology $[42,52]$.

\section{Hybrid imaging}

Based on the success of semiconductor technology, all major vendors have recently introduced PET/CT systems that offer PET with a coincidence time resolution of 200-300 ps and a spatial resolution of about $3-4 \mathrm{~mm}$. The insensitivity of these new detectors to strong magnetic fields stimulated the development of hybrid simultaneous PET/MRI systems, a modality that is now offered by almost all major vendors. Currently, PET/MRI is mostly used in the field of neurology, oncology, and pediatrics. In parallel to clinical PET/MRI, PET inserts were developed that convert standard MRI systems into hybrid scanners. The first systems were used in preclinical imaging $[38,65]$ and are now being further developed for various organs, such as breast PET/MRI and neuro-PET/MRI, to name just a few.
Recently, the first two total-body PET systems were stimulated from the Explorer consortium: the UEXPLORER from United Imaging Healthcare (Shanghai, China), which is installed at the University of California, Davis, and the PennExplorer, which has been developed by the University of Pennsylvania. Thanks to their large axial field of view, these systems offer system sensitivity up to a factor of 40 higher than today's clinical PET/CT scanners. This enables a range of novel applications, from ultralow dose PET images to dynamic imaging within sub-seconds and total-body pharmacokinetics (• Fig. 3a, b; [72]).

In SPECT, pinhole collimator technology has become the state of the art for preclinical imaging. The first clinical SPECT system for brain and large animal imaging is now available (G-SPECT, MILabs, The Netherlands), which offers a spatial resolution of down to $3 \mathrm{~mm}$ [7]. 


\section{Machine learning}

Parallel to these innovations on the system level, the field of software-based data processing is developing rapidly through the application of machinelearning methods. These algorithms provide improved and faster image reconstruction with lower image noise, real-time scatter correction, and improved spatial resolution by integrating machine-learning methods along the entire signal processing chain. For instance, a denoising convolutional neural network (DnCNN) was proposed as an iterative reconstruction framework for PET, which improves the reconstruction result both quantitatively and qualitatively (- Fig. 3c-e; [32]). For the field of detector data processing, gradient tree boosting machine-learning methods have shown a high potential for $3 \mathrm{D}$ event positioning within monolithic and semi-monolithic scintillator crystals, which may be the crystal topology of the future [43].

\section{Radioligands}

In addition to these technical developments, several promising radioligands have been developed and clinically applied in recent years. A very promising candidate is ${ }^{68} \mathrm{Ga}$-PSMA. A recent study showed that this PSMA ligand surpasses all other imaging modalities for the restaging of prostate cancer with a detection rate of $>90 \%$ for patients with prostate-specific antigen levels over $1 \mathrm{ng} / \mathrm{ml}$ [19]. Another promising PET radiotracer uses quinoline-based fibroblast activation protein inhibitors (FAPI) and targets the tumor stroma, i.e., cancerassociated fibroblasts. In this respect, ${ }^{68} \mathrm{Ga}$-FAPI PET/CT has been reported to be an alternative diagnostic method to ${ }^{18} \mathrm{~F}-\mathrm{FDG}$, allowing us to image various types of cancer, such as head and neck, colon, lung, breast, and pancreatic cancer with tumor-to-background contrast ratios equal to or even better than those of ${ }^{18}$ F-FDG [21].

\section{Immuno-PET imaging}

A further emerging area is ImmunoPET [61]. The recent clinical successes of immunotherapies require the noninvasive monitoring of the tumor immune response to therapy. ${ }^{89} \mathrm{Zr}$-Immuno-PET may play a key role in this context as it combines the specificity and affinity of antibodies with the sensitivity of PET [60, 64]. The long half-life of ${ }^{89} \mathrm{Zr}$ $(t=78.4 \mathrm{~h})$ enables measurements over longer time intervals, which is important since the accumulation of antibodies in tumors is slow and active targeting effects can only be distinguished at late time points. Several clinical trials reported on the clinical performance of Immuno-PET for a variety of tumors, such as head and neck cancer, breast cancer, B-cell lymphoma, glioblastoma, neuroendocrine tumors, non-small cell lung cancer, prostate cancer, pancreatic cancer, ovarian cancer, colorectal cancer, and renal cell carcinoma $[8,28]$.

Overall, the number of innovations in the field of nuclear medicine has been enormous in the past decade. Nuclear medicine imaging will become more precise and more quantitative in the near future, due to the use of new technologies, machine-learning methods, and novel radioligands, paving the way to new applications.

\section{Magnetic particle imaging}

Magnetic particle imaging (MPI) is a novel, tracer-based imaging modality that quantitatively visualizes super-paramagnetic iron oxide-based nanoparticles (SPIONs) in vitro and in vivo. Magnetic particle imaging exploits the non-linear magnetization function of SPIONs by applying multiple superimposed static and dynamic magnetic fields and measuring the distorted magnetic response of the particles. Currently, commercial preclinical scanners offer high sensitivity-5-20 ng(Fe), $2.8 \mu \mathrm{mol}(\mathrm{Fe}) / 1-s u b-$ millimeter spatial resolution, and high temporal resolution with up to 46 volumes per second [23]. Since MPI only shows the tracer distribution, it lacks anatomical information, which can be acquired by anatomical imaging modalities followed by image fusion. Consequently, hybrid MPI-MRI scanners have already been developed to facilitate co-registration, which speeds up the acquisition process and simplifies the workflow [20].

The high temporal resolution of MPI enables real-time imaging, making MPI capable of perfusion imaging and blood flow velocity quantification (- Fig. 4a; [67]). Thus, MPI is often discussed as a modality for diagnosing vascular diseases and for supporting vascular interventions [6, 50]. A human-sized MPI system developed for brain perfusion imaging was recently reported (- Fig. 4b; [24]). Another promising feature of MPI is the ability to obtain parameters of the SPION local environment such as temperature, viscosity, or particle mobility/binding state [47]. Knowledge of these parameters can be beneficial in applications such as magnetic hyperthermia or nanomedicine [14]. Other potential applications of MPI are cell tracking, interventional medicine, and diagnosis of inflammatory diseases and cancer $[6,50]$.

However, the clinical translation of the young modality MPI (invented 2005) is a tough challenge, since it needs to compete with other clinically established imaging methods. Upscaling to humansized scanners has just begun. Additionally, the predicted iron detection limit of MPI $-<1 \mu \mathrm{mol}(\mathrm{Fe}) / 1-$ that would outperform the sensitivity of SPION-enhanced MRI- 50 $\mu \mathrm{mol}(\mathrm{Fe}) / 1-$ is still not proven experimentally [66]. Reasons might be the lack of MPI-optimized tracers and hardware, e.g., distortions of power electronics raising the noise floor. Further enhancement of MPI performance may result from advances in reconstruction techniques and imaging sequences. Reaching the predicted detection limit would strengthen the position of MPI against the established imaging modalities. Finally, there is still an intense search for a clinical application giving it a strong unique selling point. 

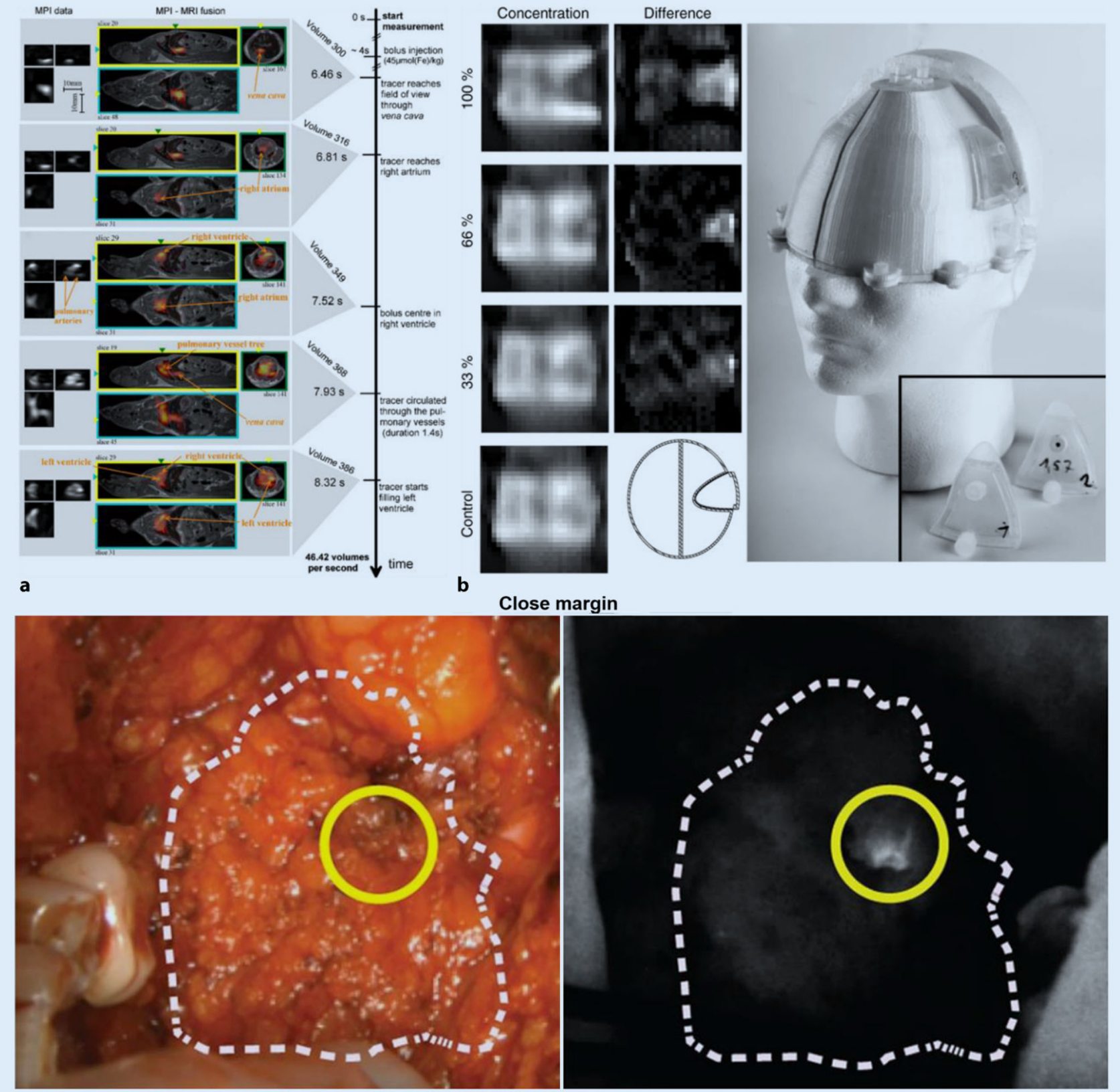

C

Deep margin of primary specimen - in situ

Fig. $4 \Delta$ a Real-time in vivo measurement of a beating mouse heart. The magnetic particle imaging $(M P I)$ images were fused with static MRI images. SPION (Resovist, Bayer Schering Pharma AG, Wuppertal, Germany) was injected into the tail vein of the mouse. The successive phases of the bolus passage can be taken from the time axis on the right. All heart chambers and parts of the vessel tree could be identified (Reprinted with permission from [67]). b Stroke phantom images acquired with the human-sized MPI for brain applications. Different grades of perfusion deficits were simulated by filling Perimag (micromod Partikeltechnologie $\mathrm{GmbH}$, Rostock, Germany) with varying iron concentrations in the fitting part that reflects the stroke. In the control case, the iron concentration was the same as in the left hemisphere $\left(965 \mathrm{ng}_{\mathrm{Fe}} \mathrm{ml}^{-1}\right)$. The stroke areas could be identified in the difference image for $100 \%, 66 \%$, and 33\% reduction. (The work is licensed under CC BY 4.0 from [24] https:// creativecommons.org/licenses/by/4.0/). c Bright field and fluorescence images of the primary lesion resection margin (dotted line) from patient with head and neck cancer, who received an i.v. injection of panitumumab-IRDye800CW. The fluorescence image shows some remaining fluorescence (yellow circle), which was proven to be a close margin (distance from primary lesion to resection margin $<5 \mathrm{~mm}$ ). (This research was originally published in JNM. Van Keulen S., et al. The Clinical Application of Fluorescence-Guided Surgery in Head and Neck Cancer.J Nucl Med. 2019;60:758-763@SNMMI [59] under CC BY 4.0 https:// creativecommons.org/licenses/by/4.0/.) 


\section{Optical and photoacoustic imaging}

Optical imaging techniques rely on photons in the visible or near-infrared wavelength range, which interact with tissue, and are captured by a camera. Fluorescence and photoacoustic imaging involve light sources external to the tissue, whereas bioluminescence imaging makes use of photons generated by enzymatically catalyzed chemical reactions, as is known from fireflies. Bioluminescence imaging is of less relevance for clinical imaging; hence it is not discussed in the following.

\section{Fluorescence imaging}

The penetration depth of only a few millimeters limits applications of fluorescence imaging to superficial structures, such as skin lesions or tissues accessible by endoscopes. Narrow-band illumination sources and optical filters enable acquisition of multiple channels for improved discrimination of tissue types, and such multispectral endoscopic systems were recently shown to enable higher sensitivity and specificity for surveillance of Barrett's esophagus and polyp detection in colonoscopy [3, 30]. Fluorescent contrast agents can be applied by spraying or by injection to achieve highly specific contrast with low background to better find or differentiate neoplastic lesions and identify positive resection margins during image-guided surgery [45]. For example, an NIR-labeled EGFR-binding antibody was recently shown to improve the outcome of surgical resections of head and neck cancers (- Fig.4c; [59]). Commercially available imaging systems have stimulated the development and clinical approval of additional fluorescent probes, including antibody fragments with faster background clearance and activatable probes that do not require washing after spraying onto the tissue of interest [15].

\section{Photoacoustic approaches}

Photoacoustic devices employ pulsed light and acquire acoustic echoes based on the thermoelastic effect and reconstruct a 3D image of the optical absorption. Innate chromophores such as oxygenated and deoxygenated hemoglobin, lipids, and melanin are abundant and can be discriminated using multispectral photoacoustic approaches, supporting potential screening applications for breast cancer, skin melanoma, and psoriatic skin lesions [4]. A prospective multicenter study showed that many suspicious breast masses could be correctly downgraded after photoacoustic imaging with native contrast, potentially reducing the number of biopsies of benign lesions [41]. Multispectral photoacoustic imaging combined with indocyanine green was able to assess the metastasis state of sentinel lymph nodes in melanoma patients [55]. Further applications of photoacoustic imaging may be enabled by improved contrast agents with extended blood circulation, higher absorption, specific absorption spectra, or molecular targeting [54].

\section{Advances in ultrasound}

\section{Ultrafast Doppler ultrasound and sheer wave elastography}

Ultrasound enables the visualization and quantification of blood flow in larger blood vessels. However, to date, microvessels could hardly be assessed without contrast agents (microbubbles). This has changed with the development of ultrafast Doppler methods enabling frame rates of several thousand hertz, which is more than 100-fold higher than what conventional ultrasound systems offer. Ultrafast Doppler ultrasound was successfully applied to visualize microvascular anatomy and function in the brain of human newborns and it was able to depict different sleep states as well as dynamics of neonatal seizures (• Fig. 5a; [17]). Furthermore, it also facilitated the development of sheer wave elastography (SWE), a real-time method to visualize and quantify the stiffness of tissues (- Fig. $5 b$ ). Sheer wave elastography proved promising for the detection of the degradation of tendons and neoplastic lesions, although the clinically most advanced application is characterization of liver fibrosis [35], for which large clinical trials have already been performed.

\section{Ultrasound localization microscopy}

Another important innovation is the development of ultrasound localization microscopy [13]. Here, injected microbubbles are followed within the image plane and the resulting tracks are indicated in the B-mode images. Since the tracks can be drawn significantly smaller than the voxels, the vascular representation exceeds the resolution of the ultrasound transducers. Two different strategies were suggested, both being currently implemented into commercial ultrasound devices [13]. One measures the motion of the bubbles with ultrafast ultrasound (uULM), the other uses motion models to determine probabilities of microbubbles to have moved to a certain position in subsequent frames. The latter method, called "motion model ultrasound localization microscopy" (mULM), was the first to be applied in humans and proved promising to detect neovasculature in breast cancers and to assess therapy responses (• Fig. 5c; [46]).

\section{Three-dimensional data acquisition}

Multiple new tissue characteristics such as velocities in individual microvessels or flow directions can be quantified that were not accessible so far, broadening perspectives for radiomic analyses. It can be expected that the method can be improved significantly by the introduction of matrix ultrasound transducers facilitating true 3D image acquisition. Such transducers have been implemented in endoscopic ultrasound devices and recently also in transducers for abdominal and breast imaging by several companies. Three-dimensional data can be used to get a more detailed display of vascular structure, to perform motion correction, and to improve reproducibility, the latter still being a prominent limitation of ultrasound.

Three-dimensional data acquisition may also support the implementation of molecular ultrasound imaging that uses 

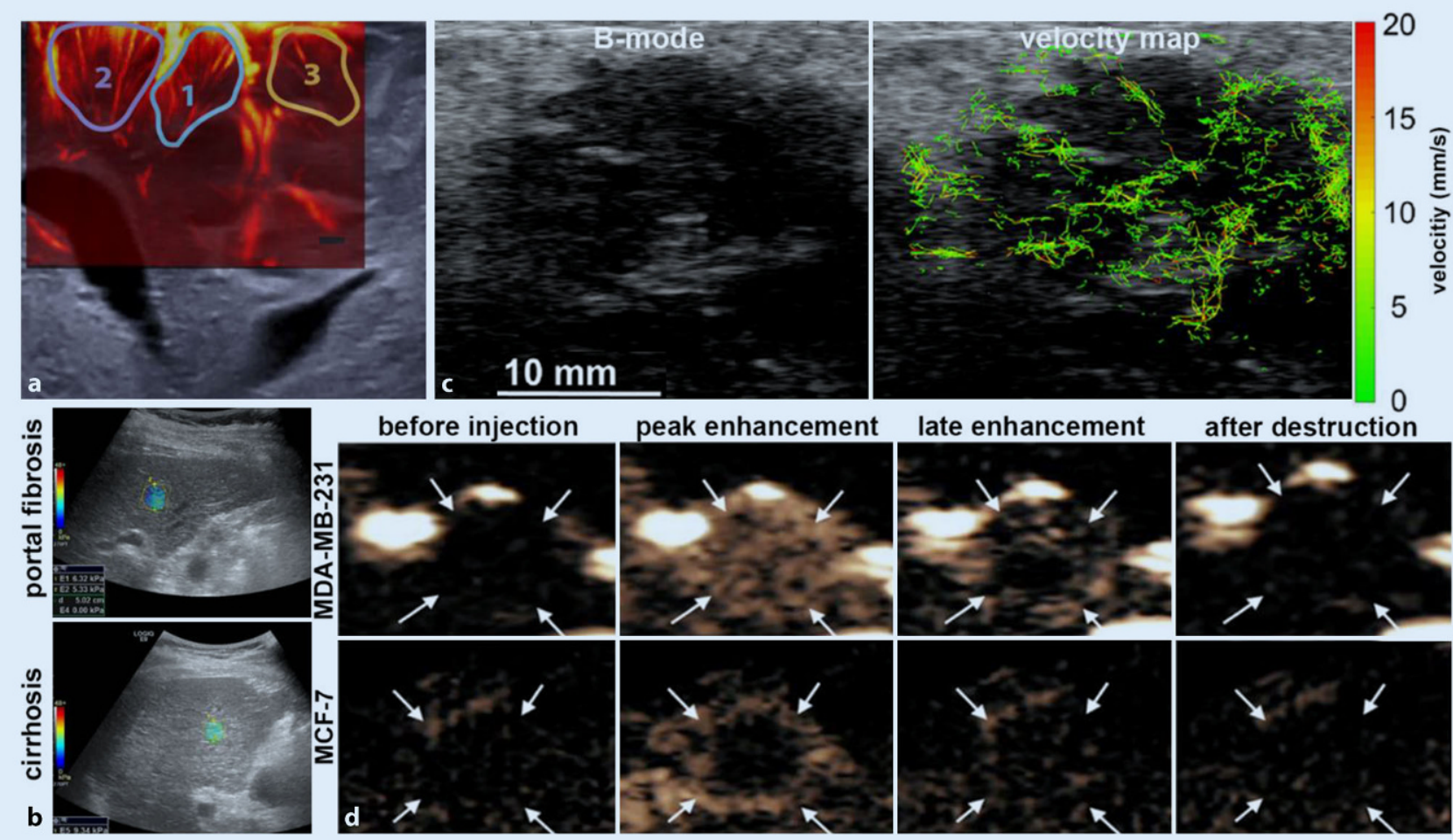

after destruction
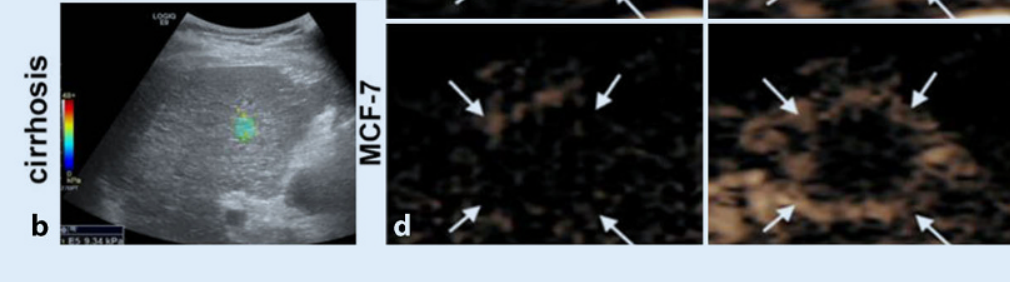

Fig. $5 \Delta$ a Ultrafast Doppler image of a human neonate brain overlaid on a B-mode image. Colored circles represent cortical regions of interest within parietal, cortical, and subcortical regions. (Reprinted with permission from [17]). b Sheer wave elastography reliably depicts liver fibrosis as demonstrated in the (color coded) liver images of patients with periportal fibrosis (fibrosis grade 2) and cirrhosis (fibrosis grade 4). (The work is licensed under CC BY 4.0 from [35], under https:// creativecommons.org/licenses/by/4.0/.). CB-mode image of a human breast cancer (left) and the according super-resolution ultrasound image (mULM; right) indicating velocities in individual microvessels. (The work is licensed under CC BY 4.0 from [46], https://creativecommons.org/licenses/by/4.0/.). d Ability to distinguish high (MDA-MB-231) and low malignant breast cancers (MCF-7) in mice by molecular ultrasound imaging. The peak enhancement images indicate a lower vascularization of the MCF-7 tumors, while the late enhancement images confirm higher binding of VEGFR2-targeted microbubbles to the highly malignant MDA-MB-231 tumors and thus higher angiogenic activity. The destructive pulse was applied to indicate that the signals in the late enhancement images were not related to microbubbles circulating in the blood pool. (Reprinted with permission from [10])

microbubbles targeted against inflammatory or angiogenic vasculature. Here, 3D matrix transducers were applied to monitor antiangiogenic therapy response in colon cancer using the VEGFR2-targeted microbubble BR55 [63]. BR55 is the first molecularly targeted ultrasound contrast agent that has been evaluated in patients. Various preclinical and clinical studies proved its value in detecting prostate, breast, and ovarian cancer (• Fig. 5d; [10]). In addition, in preclinical studies, it faithfully assessed dysplastic transformation of liver tissue [25] and was superior to standard contrast-enhanced ultrasound methods in differentiating breast cancers of different malignity and in monitoring antiangiogenic therapy responses [5]. Other microbubbles with high potential for clinical translation are targeted against $\mathrm{P}$ - or E-selectin as well as

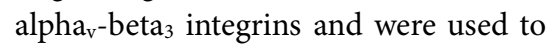
monitor acute and chronic inflammatory bowel disease as well as cardiovascular pathologies.

However, despite this encouraging progress in ultrasound technology, its limited reproducibility and user dependence remain a significant limitation. Great efforts should be made in this regard, e.g., by improving and standardizing the training of physicians, developing digital assist systems (e.g., guiding the scanning procedure or supporting lesion detection), and implementing ultrasound systems with true 3D imaging capabilities.

\section{Radiomics and comprehensive diagnostics}

For many years, new contrast agents and imaging methods were the primary drivers to improve disease diagnosis. Currently, advanced analytical tools including artificial intelligence have opened up new avenues to make use of unused and unknown information hidden in the imaging data.

In this context, radiomics is a new field in radiology that extracts quantitative imaging features from medical images to describe the phenotype of lesions, and associates it with biological characteristics, therapeutic responses, or clinical outcome (• Fig. 6). 


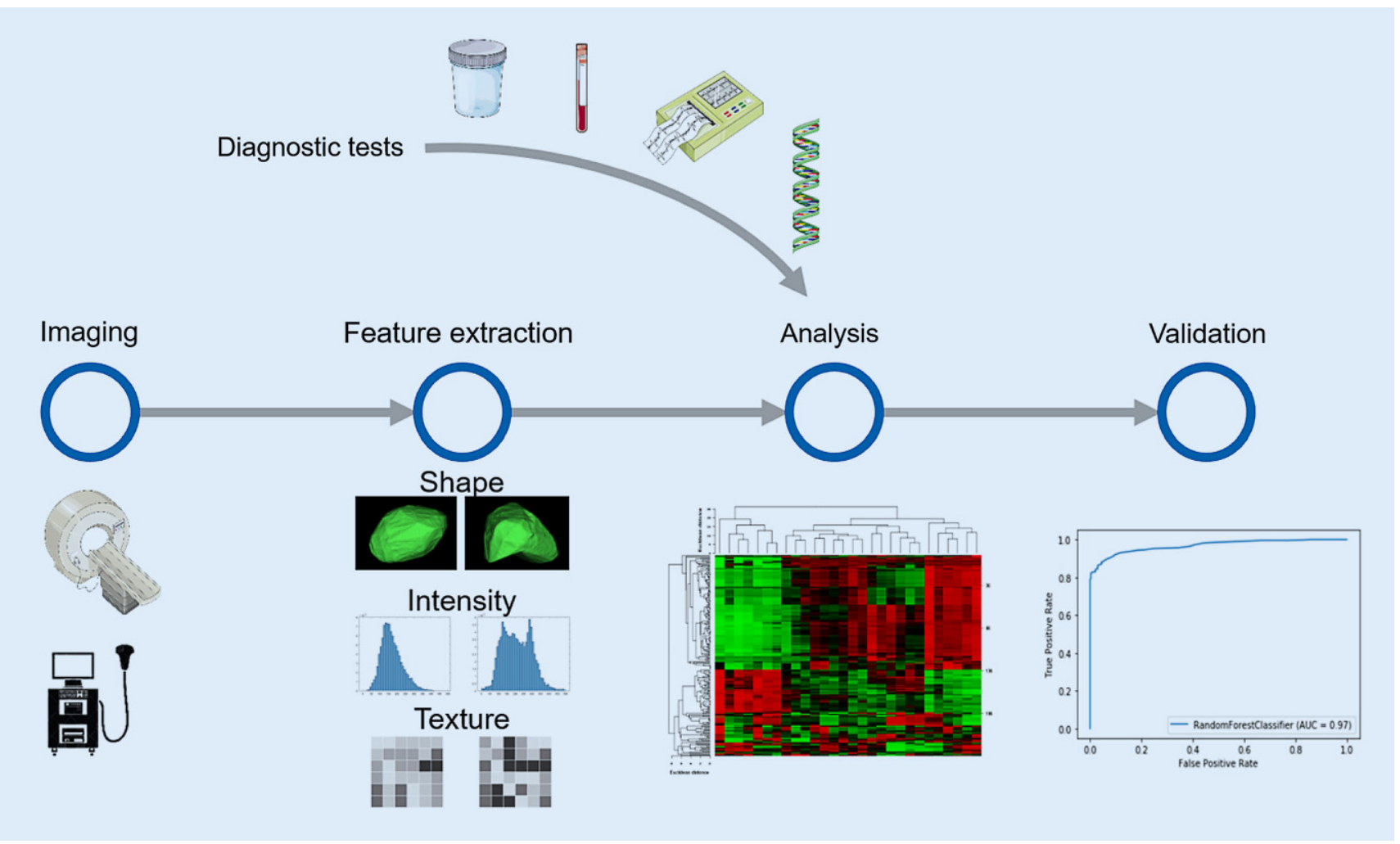

Fig. $6 \Delta$ Schematic representation of a radiomic pipeline. Diagnostic imaging is followed by lesion segmentation and feature extraction. Classically, shape, intensity, and textural parameters are extracted, but can be extended by functional and molecular parameters. The data analysis can either be based on imaging data, which is compared with clinical data and gene expression, or be combined with these additional clinical parameters, to develop decision support systems for precision medicine and therapy monitoring

The imaging features are frequently based on intensity, shape, and textural characteristics of the lesion; however, they can also contain functional or molecular imaging information $[1,22,57]$. For example, Aerts and colleagues found a radiomic signature in CT data from patients with lung and head-and-neck cancer, describing the intratumoral heterogeneity, and showing a good prognostic potential in a validation cohort [1]. Additionally, they proved that quantitative imaging parameters and clinical data contained complementary information, and their combined analysis led to better results in comparison with the individual data alone. Radiomic analyses are not limited to the analysis of images of a single time point, but can also be compared between different time points, which is known as "delta-radiomics" [34].

The quality and repeatability of radiomic studies do not depend only on the number of available data, but also on other aspects, such as scanner model, image acquisition protocol, image reconstruction, and lesion segmentation [39, 73]. Hence, radiomic pipelines need to be developed, and, ideally, seamlessly integrated into the clinical workflow, in order to standardize the process from image acquisition until lesion characterization and classification, to reduce inter-operator variability, and to ensure generalizability. This includes the development of semi-automated segmentation and feature extraction algorithms and the analysis of data from multi-center studies, to identify or generate features which are robust when using different scanners and scan protocols. The final lesion evaluation task is solved by machine-learning algorithms, performing a regression or classification task. Various algorithms from classic support vector machines and decision trees to deep-learning classifiers are used. The latter attracted much attention in the past few years as they can combine some tasks in a radiomic pipeline, such as automated feature extraction and performance of predictions or classifications.

However, there is still uncertainty about the ideal algorithm, which strongly depends on the medical question and the data. Eventually, the combination of different algorithms might prove to be superior, as shown by Diamant et al., who combined a classic radiomics model and a deep-learning model [18]. Even though some of the layers of the neural network explicitly recognized radiomic features, which were also found to be predictive by the classic approach, the prediction for the presence of distant metastasis in patients with head-andneck cancer was significantly improved when both models were combined.

Although more and more algorithms are developed for solving specific tasks-sometimes with a comparable performance to radiologists-only a few artificial-intelligence-based solutions are approved for clinical use. A major limitation of many studies is their limited 
generalizability. Since the patient cohort used to train and test the algorithm often originates from a single site only, its performance on new data originating from different sites is often worse. Focusing on sampling a truly representative cohort of the population from multiple sites for the training process of the algorithm can already help to increase its robustness and generalizability, since the variability in the population, as well as the variability of the imaging devices used, better resembles the real-world scenario.

Beside these more technical challenges, regulatory aspects must also be adapted to provide guidelines for the development of artificial-intelligencebased solutions, e.g., how to deal with machine-learning-based algorithms that are continuously learning. Eventually, these new developments will support radiologists in their daily routine, taking over some tiring and redundant work and help to better standardize structured radiologic reporting. Once certain classifier models have proven their value in prospective studies, relevant imaging biomarkers can be automatically integrated in the radiologic reports. This would simplify the integration of relevant imaging biomarkers and other available clinical data in decision support systems to improve diagnosis and personalize therapy.

\section{Future perspectives}

The technological developments mentioned here will significantly extend the range of morphological, functional, and molecular information that can be obtained by imaging. This will require that radiologists deepen their knowledge in pathophysiology and molecular mechanisms of diseases and explore how these affect their diagnostic findings. They also need to investigate how the array of diagnostic biomarkers obtained will interact and change after therapeutic interventions.

Comprehensive diagnostics is the key to precision medicine, and its potential has been shown by multiple publications. However, without proper solutions for data integration in clinical routine, digital patient files, and (inter- )national E-health infrastructures, it will not become a clinical reality [9]. Profound knowledge of information technology infrastructures, data formats, and interfaces must be acquired and legal prerequisites for data ownership, data sharing, and protection of patients' privacy clarified. Collaborations between different diagnostic specialties, i.e., radiology, nuclear medicine, pathology, and laboratory medicine need to be intensified since the integrated use of cross-disciplinary information will make the borders between the different diagnostic medical disciplines more flexible [31]. This may require a significant adaptation of the professional education of radiologists. In this context, effort should be taken to understand the principles and potential pitfalls of computer-assisted image analysis, radiomics, and deep-learning algorithms applied to generate powerful diagnostic assays.

\section{Practical conclusion}

- Imaging modalities have undergone rapid developments in recent decades and the application area of current devices is continually increasing.

- With the rise of deep learning, radiomics, and comprehensive data analysis, a new era of diagnostics has been ushered in. Established imaging methods are now complemented by new applications such as magnetic particle imaging (MPI), optical imaging, and photoacoustics.

- The potential of imaging to describe pathophysiological relationships in ever-increasing detail, both at wholebody and tissue level, can be used to better understand the mechanistic effect of drugs, to preselect patients to therapies, and to improve monitoring of therapy success.

- The use of interdisciplinary integrated diagnostics will greatly change but also enrich the profession of radiologists.

\section{Corresponding address}

Professor Dr. med. F. Kiessling

Institute for Experimental Molecular Imaging, Medical Faculty, RWTH Aachen International University

Forckenbeckstraße 55, 52074 Aachen, Germany fkiessling@ukaachen.de

\section{Compliance with ethical guidelines}

Conflict of interest. F. Gremse is the founder of Gremse-IT GmbH, Aachen, Germany, and a consultant for MILabs B.V., Utrecht, The Netherlands. V. Schulz is the founder and CEO of HYPERION Hybrid Imaging Systems GmbH, Aachen, Germany. F. Kiessling was a consultant for Bracco and holds patents and has cooperations with Fuji Visualsonics, Bayer, and Roche. Furthermore, some of his work on BR55 was financed by Bracco and Merck Darmstadt. B. Theek, T. Nolte, D. Pantke, and F. Schrank declare that they have no competing interests.

For this article no studies with human participants or animals were performed by any of the authors. All studies performed were in accordance with the ethical standards indicated in each case.

The supplement containing this article is not sponsored by industry.

\section{References}

1. Aerts HJ, Velazquez ER, Leijenaar RT et al (2014) Decoding tumour phenotype by noninvasive imaging using a quantitative radiomics approach. NatCommun 5:4006

2. Aggarwal R, Vigneron DB, Kurhanewicz J (2017) Hyperpolarized 1-[(13)C]-pyruvate magnetic resonance imaging detects an early metabolic response to androgen ablation therapy in prostate cancer. Eur Urol 72:1028-1029

3. Atkinson NSS, Ket S, Bassett $P$ et al (2019) Narrow-band imaging for detection of neoplasia at colonoscopy: a meta-analysis of data from individual patients in randomized controlled trials. Gastroenterology 157:462-471

4. Attia ABE, Balasundaram G, Moothanchery $M$ et al (2019) A review of clinical photoacoustic imaging: current and future trends. Photoacoustics 16:100144

5. Baetke SC, Rix A, Tranquart F et al (2016) Squamous cell carcinoma xenografts: use of VEGFR2targeted microbubbles for combined functional and molecular US to monitor antiangiogenic therapy effects. Radiology 278:430-440

6. Bakenecker AC, Ahlborg M, Debbeler Cet al (2018) Magnetic particle imaging in vascular medicine. Innov Surg Sci 3:179-192

7. Beekman FJ, Van der Have F, Goorden MC et al (2015) G-SPECT-I: a full ring high sensitivity and ultra-fast clinical molecular imaging system with $<3 \mathrm{~mm}$ resolution. Eur J Nucl Med Mol Imaging 42:S209-S209

8. Borjesson PK, Jauw YW, Boellaard R et al (2006) Performance of immuno-positron emission tomography with zirconium-89-labeled chimeric 
monoclonal antibody U36 in the detection of lymph node metastases in head and neck cancer patients. Clin Cancer Res 12:2133-2140

9. Bukowski M, Farkas R, Beyan O et al (2020) Implementation of ehealth and $\mathrm{Al}$ integrated diagnostics with multidisciplinary digitized data: are we ready from an international perspective? Eur Radiol. https://doi.org/10.1007/s00330-02006874-x

10. Bzyl J, Lederle W, Rix A et al (2011) Molecular and functional ultrasound imaging in differently aggressive breast cancer xenografts using two novel ultrasound contrast agents (BR55 and BR38). EurRadiol 21:1988-1995

11. Chen F, Taviani V, Malkiel I et al (2018) Variabledensity single-shot fast spin-echo MRI with deep learning reconstruction by using variational networks. Radiology 289:366-373

12. Chen H, Zhang Y, Kalra MK et al (2017) Low-dose CT with a residual encoder-decoder convolutional neural network. IEEE Trans Med Imaging 36:2524-2535

13. Christensen-Jeffries K, Couture O, Dayton PA et al (2020) Super-resolution ultrasound imaging. Ultrasound Med Biol 46(4):865-891. https://doi. org/10.1016/j.ultrasmedbio.2019.11.013

14. Dasgupta A, Biancacci I, Kiessling F et al (2020) Imaging-assisted anticancer nanotherapy. Theranostics 10:956-967

15. Debie P, Hernot S (2019) Emerging fluorescent molecular tracers to guide intra-operative surgical decision-making. Front Pharmacol 10:510

16. Dehkharghani S, Qiu D, Albin LS et al (2015) Dose reduction in contrast-enhanced cervical MR angiography: field strength dependency of vascular signal intensity, contrast administration, and arteriographic quality. AJR Am J Roentgenol 204:W701-706

17. Demene C, Baranger J, Bernal M et al (2017) Functional ultrasound imaging of brain activity in humannewborns. SciTransIMed 9(411):eaah6756. https://doi.org/10.1126/scitranslmed.aah6756

18. Diamant A, Chatterjee A, Vallieres M et al (2019) Deep learning in head \& neck cancer outcome prediction. Sci Rep 9:2764

19. Eiber M, Maurer T, Souvatzoglou M et al (2015) Evaluation of hybrid 68ga-PSMA ligand PET/CT in 248 patients with biochemical recurrence after radical prostatectomy. J Nucl Med 56:668-674

20. Franke J, Heinen U, Lehr H et al (2016) System characterization of a highly integrated preclinical hybrid MPI-MRI scanner. IEEE Trans Med Imaging 35:1993-2004

21. GieselFL,KratochwilC, LindnerTetal(2019)(68)gaFAPI PET/CT: biodistribution and preliminary dosimetry estimate of 2 DOTA-containing FAPtargeting agents in patients with various cancers. JNucl Med 60:386-392

22. Gillies RJ, Kinahan PE, Hricak H (2016) Radiomics: images are more than pictures, they are data. Radiology 278:563-577

23. Graeser M, Knopp T, Szwargulski P et al (2017) Towards picogram detection of superparamagnetic iron-oxide particles using a gradiometric receive coil. Sci Rep 7:6872

24. Graeser M, Thieben F, Szwargulski P et al (2019) Human-sized magnetic particle imaging for brain applications. Nat Commun 10(1):1936. https://doi. org/10.1038/s41467-019-09704-x

25. Grouls C, Hatting M, Rix A et al (2013) Liver dysplasia: US molecular imaging with targeted contrast agent enables early assessment. Radiology 267:487-495
26. Hata A, Yanagawa M, Honda O et al (2018) Effect of matrix size on the image quality of ultra-highresolution $C T$ of the lung: comparison of $512 x$ 512,1024 × 1024, and $2048 \times 2048$. Acad Radiol 25:869-876

27. Hu Z, Jiang C, Sun F et al (2019) Artifact correction in low-dose dental CT imaging using Wasserstein generative adversarial networks. Med Phys 46:1686-1696

28. Jauw YW, Menke-van der Houven van Oordt CW Hoekstra OS et al (2016) Immuno-positron emission tomography with zirconium-89-labeled monoclonal antibodies in oncology: what can we learn from initial clinical trials? Front Pharmacol 7:131

29. Jones KM, Pollard AC, Pagel MD (2018) Clinical applications of chemical exchange saturation transfer (CEST) MRI. J Magn Reson Imaging 47:11-27

30. Kato M, Goda K, Shimizu Y et al (2017) Image assessment of Barrett's esophagus using the simplified narrow band imaging classification. J Gastroenterol 52:466-475

31. Kiessling F (2018) The changing face of cancer diagnosis: from computational image analysis to systems biology. Eur Radiol 28:3160-3164

32. Kim K, Wu D, Gong K et al (2018) Penalized PET reconstruction using deep learning prior and local linear fitting. IEEE Trans Med Imaging 37:1478-1487

33. Kim M, Torrealdea F, Adeleke S et al (2019) Challenges in glucoCEST MR body imaging at 3 Tesla. Quant Imaging Med Surg 9:1628-1640

34. Lambin P, Leijenaar RTH, Deist TM et al (2017) Radiomics: the bridge between medical imaging and personalized medicine. Nat Rev Clin Oncol 14:749-762

35. Lee SM, Lee JM, Kang HJ et al (2017) Liver fibrosis staging with a new 2D-shear wave elastography using comb-push technique: applicability, reproducibility, and diagnostic performance. PLoS ONE 12:e177264

36. Lundervold AS, Lundervold A (2019) An overview of deep learning in medical imaging focusing on MRI.ZMed Phys 29:102-127

37. Ma D, Gulani V, Seiberlich N et al (2013) Magnetic resonance fingerprinting. Nature 495:187-192

38. Mackewn JE, Lerche CW, Weissler B et al (2015) PET performance evaluation of a pre-clinical siPMbased MR-compatible PET scanner. IEEE Trans Nuc Sci 62:784-790

39. Mackin D, Fave X, Zhang L et al (2015) Measuring computed tomography scanner variability of radiomics features. Invest Radiol 50:757-765

40. Mechlem K, Ehn S, Sellerer T et al (2018) Joint statistical iterative material image reconstruction for spectral computed tomography using a semiempirical forward model. IEEE Trans Med Imaging 37:68-80

41. Menezes GLG, Pijnappel RM, Meeuwis C et al (2018) Downgrading of breast masses suspicious for cancer by using optoacoustic breast imaging Radiology 288:355-365

42. Müller F, Schug D, Hallen P et al (2018) Gradient tree boosting-based positioning method for monolithic scintillator crystals in positron emission tomography. IEEE Trans Radiat Plasma Med Sci 2:411-421

43. Müller F, Schug D, Hallen P et al (2018) A novel DO positioning algorithm for monolithic scintillator crystals in PET based on gradient tree boosting. IEEE Trans Radiat Plasma Med Sci 3:465-474

44. Neuhaus E, Weiss K, Bastkowski R et al (2019) Accelerated aortic 4D flow cardiovascular magnetic resonance using compressed sensing: applicability, validation and clinical integration. J Cardiovasc Magn Reson 21:65

45. Newton AD, Predina JD, Nie S et al (2018) Intraoperative fluorescence imaging in thoracic surgery. J Surg Oncol 118:344-355

46. Opacic T, Dencks S, Theek B et al (2018) Motion model ultrasound localization microscopy for preclinical and clinical multiparametric tumor characterization. NatCommun 9:1527

47. Pantke D, Holle N, Mogarkar A et al (2019) Multifrequency magnetic particle imaging enabled by a combined passive and active drive field feedthrough compensation approach. Med Phys 46:4077-4086

48. Pourmorteza A, Symons R, Sandfort V et al (2016) Abdominal imaging with contrast-enhanced photon-counting CT: first human experience. Radiology 279:239-245

49. Qin C, Schlemper J, Caballero J et al (2019) Convolutional recurrent neural networks for dynamic MR image reconstruction. IEEE Trans Med Imaging 38:280-290

50. Rahmer J, Wirtz D, Bontus C et al (2017) Interactive magnetic catheter steering with 3-D real-time feedback using multi-color magnetic particle imaging. IEEE Trans Med Imaging 36:1449-1456

51. Rauch T, Rieger J, Pelzer G et al (2020) Discrimination analysis of breast calcifications using $x$-ray dark-field radiography. Med Phys. https://doi.org/ 10.1002/mp.14043

52. Schug D, Lerche C, Weissler B et al (2016) Initial PET performance evaluation of a preclinical insert for PET/MRI with digital SiPM technology. Phys Med Biol 61:2851-2878

53. Si-Mohamed S, Bar-Ness D, Sigovan $M$ et al (2018) Multicolour imaging with spectral photoncounting CT: a phantom study. Eur Radiol Exp 2:34

54. Steinberg I, Huland DM, Vermesh $O$ et al (2019) Photoacoustic clinical imaging. Photoacoustics 14:77-98

55. Stoffels I, Morscher S, Helfrich I et al (2015) Metastatic status of sentinel lymph nodes in melanoma determined noninvasively with multispectral optoacoustic imaging. Sci Transl Med 7:317ra199

56. Symons R, Cork TE, Sahbaee Pet al (2017) Low-dose lung cancer screening with photon-counting $\mathrm{CT}$ : a feasibility study. Phys Med Biol 62:202-213

57. TheekB,Opacic T,MagnuskaZetal (2018) Radiomic analysis of contrast-enhanced ultrasound data. Sci Rep 8:11359

58. UeckerM,Zhang S, VoitDetal (2010) Real-time MRI at a resolution of $20 \mathrm{~ms}$. NMRBiomed 23:986-994

59. van Keulen S, Nishio N, Fakurnejad Set al (2019) The clinical application of fluorescence-guided surgery in head and neck cancer. J Nucl Med 60:758-763

60. Verel I, Visser GW, Boellaard R et al (2003) 89Zr immuno-PET: comprehensive procedures for the production of $89 \mathrm{Zr}$-labeled monoclonal antibodies. J Nucl Med 44:1271-1281

61. Verel I, Visser GW, van Dongen GA (2005) The promise of immuno-PET in radioimmunotherapy. JNucl Med 46(1):164S-171S

62. Walker-Samuel S, Ramasawmy R, Torrealdea F et al (2013) In vivo imaging of glucose uptake and metabolism in tumors. Nat Med 19:1067-1072

63. Wang H, Lutz AM, Hristov D et al (2017) Intraanimal comparison between three-dimensional molecularly targeted US and three-dimensional dynamic contrast-enhanced US for early antiangiogenic treatment assessment in colon cancer. Radiology 282:443-452 
64. WeiW, RosenkransZT, Liu Jetal (2020) ImmunoPET: concept, design, and applications. Chem Rev. https://doi.org/10.1021/acs.chemrev.9b00738

65. Weissler B, Gebhardt P, Dueppenbecker PM et al (2015) A digital preclinical PET/MRI insert and initial results. IEEE Trans Med Imaging 34:2258-2270

66. Weizenecker J, Borgert J, Gleich B (2007) A simulation study on the resolution and sensitivity of magnetic particle imaging. Phys Med Biol 52:6363-6374

67. Weizenecker J, Gleich B, Rahmer J et al (2009) Three-dimensional real-time in vivo magnetic particle imaging. Phys Med Biol 54:L1-L10

68. Wieczorek M, Schaff F, Jud C et al (2018) Brain connectivity exposed by anisotropic X-ray darkfield tomography. Sci Rep 8:14345

69. Willer K, Fingerle AA, Gromann LB et al (2018) X-ray dark-field imaging of the human lung - a feasibility study on a deceased body. PLoSONE 13:e204565

70. Wu B, Warnock G, Zaiss M et al (2016) An overview of CEST MRI for non-MR physicists. EJNMMI Phys $3: 19$

71. Wu D, Kim K, El Fakhri G et al (2017) Iterative low-dose $\mathrm{CT}$ reconstruction with priors trained by artificial neural network. IEEE Trans Med Imaging 36:2479-2486

72. Zhang X, Cherry SR, Xie Z et al (2020) Subsecond total-body imaging using ultrasensitive positron emission tomography. Proc Natl Acad Sci U S A 117:2265-2267

73. Zhao B, Tan Y, Tsai WY et al (2016) Reproducibility of radiomics for deciphering tumor phenotype with imaging. Sci Rep 6:23428

74. Zhu B, Liu JZ, Cauley SF et al (2018) Image reconstruction by domain-transform manifold learning. Nature 555:487-492 